\title{
FAMILIES OF LINEAR DIFFERENTIAL EQUATIONS RELATED TO THE SECOND PAINLEVÉ EQUATION
}

\author{
MARIUS VAN DER PUT \\ Department of Mathematics, University of Groningen \\ P.O. Box 800, 9700 AV Groningen, the Netherlands \\ E-mail: mvdput@math.rug.nl
}

\begin{abstract}
This paper is a sequel to $\mathrm{vdP}-\mathrm{Sa}$ and $\mathrm{vdP}$. The two classes of differential modules $(0,-, 3 / 2)$ and $(-,-, 3)$, related to PII, are interpreted as fine moduli spaces. It is shown that these moduli spaces coincide with the Okamoto-Painlevé spaces for the given parameters. The geometry of the moduli spaces leads to a proof of the Painleve property for PII in standard form and in the Flaschka-Newell form. The Bäcklund transformations, the rational solutions and the Riccati solutions for PII are derived from these moduli spaces.
\end{abstract}

Introduction. It is well known that isomonodromic families of linear differential equations induce solutions of Painlevé equations. The families under consideration are, a priori, given by a set $\mathbf{S}$ of differential modules $M$ over $\mathbb{C}(z)$ with $\operatorname{dim} M=2, \Lambda^{2} M$ is the trivial module and with prescribed singular points and their Katz invariants. A systematic study of these families and the corresponding monodromy spaces is the theme of [vdP-Sa]. In $\operatorname{vdP}$ a detailed study of two of these families, namely $(-,-, 5 / 2)$ and $(1 / 2,-, 1 / 2)$, is presented and their relation to Okamoto's geometric approach to Painlevé equations is clarified. In the References we have tried to select some papers out of the extensive literature related to our work. We refer to papers $\mathrm{vdP}-\mathrm{Sa}, \mathrm{vdP}$ as background for the present paper.

Each family has its own story. The first family studied here is denoted by $(0,-, 3 / 2)$ and corresponds to modules $M$ as above with two singular points, namely 0 and $\infty$. The point 0 is regular singular and $\infty$ is irregular singular with Katz invariant $3 / 2$. The generalized eigenvalues at $\infty$ are normalized to $\pm w$ with $w=z^{3 / 2}+\frac{t}{2} z^{1 / 2}$. The monodromy space $\mathcal{R}$ is given by the formal monodromy and the three Stokes maps,

2010 Mathematics Subject Classification: Primary 14D20; Secondary 14D22, 34M55.

Key words and phrases: moduli space for linear connections, irregular singularities, Stokes matrices, monodromy spaces, isomonodromic deformations, Painlevé equations.

The paper is in final form and no version of it will be published elsewhere. 
represented by the matrices

$$
\left(\begin{array}{cc}
0 & -1 \\
1 & 0
\end{array}\right),\left(\begin{array}{ll}
1 & 0 \\
a_{1} & 1
\end{array}\right),\left(\begin{array}{cc}
1 & a_{2} \\
0 & 1
\end{array}\right),\left(\begin{array}{cc}
1 & 0 \\
a_{3} & 1
\end{array}\right) .
$$

Thus $\mathcal{R}$ has coordinate ring $\mathbb{C}\left[a_{1}, a_{2}, a_{3}\right]$. The topological monodromy at $\infty$ is the product of these matrices (in this order) and the trace $s$ of the topological monodromy defines the affine parameter space $\mathcal{P}$ with coordinate ring $\mathbb{C}[s]$. The map $\mathcal{R} \rightarrow \mathcal{P}$, given by $\left(a_{1}, a_{2}, a_{3}\right) \mapsto s$, makes $\mathcal{R}$ into a family of affine cubic surfaces $\mathcal{R}(s)$, given by the equation $a_{1} a_{2} a_{3}+a_{1}-a_{2}+a_{3}+s=0$, for $s \in \mathcal{P}=\mathbb{C}$. The singularities of the fibers $\mathcal{R}(s)$ occur only for the resonant case $\theta \in \mathbb{Z}$, where $\pm \theta / 2$ are the local exponents at $z=0$. Since $s=e^{\pi i \theta}+e^{-\pi i \theta}$, this corresponds to $s= \pm 2$. For $s=2$ one finds the singular point $\left(a_{1}, a_{2}, a_{3}\right)=(-1,1,-1)$ and for $s=-2$ one has the singular point $(1,-1,1)$.

The aim of this paper is to provide $\mathbf{S}$ with a structure of algebraic variety, by constructing a fine moduli space $\mathcal{M}$ (for families of the type under consideration) with $\mathbf{S}=\mathcal{M}(\mathbb{C})$. Further $\mathcal{M}$ is identified with the Okamoto-Painlevé space for PII in the Flaschka-Newell form. The singular points of the fibers of $\mathcal{R}$ for $s= \pm 2$ are resolved by a 'level structure' added to the moduli problem. The Painlevé property is deduced for PII in the Flaschka-Newell form.

The second family $\mathbf{S}$ is denoted by $(-,-, 3)$. The modules $M$ belonging to $(-,-, 3)$ have only one singular point namely $\infty$. The Katz invariant of $\infty$ is 3 and the generalized eigenvalues at $\infty$ are normalized to $\pm\left(z^{3}+\frac{t}{2} z\right)$. The monodromy space $\mathcal{R}$ is obtained from the formal monodromy and the six Stokes maps, given by the matrices

$$
\left(\begin{array}{ll}
\alpha & 0 \\
0 & \frac{1}{\alpha}
\end{array}\right),\left(\begin{array}{ll}
1 & 0 \\
b_{1} & 1
\end{array}\right),\left(\begin{array}{rr}
1 & b_{2} \\
0 & 1
\end{array}\right), \cdots,\left(\begin{array}{rr}
1 & b_{6} \\
0 & 1
\end{array}\right) .
$$

The topological monodromy, which is the identity, is the product of the above matrices. This yields a set of relations for $\alpha$ and the $b_{*}$. Further one has to divide by the action (by conjugation) of $\mathbb{G}_{m}=\left\{\left(\begin{array}{ll}\lambda & 0 \\ 0 & 1\end{array}\right) \mid \lambda \in \mathbb{C}^{*}\right\}$. The affine parameter space $\mathcal{P}$ has coordinate ring $\mathbb{C}\left[\alpha, \alpha^{-1}\right]$. The morphism $\mathcal{R} \rightarrow \mathcal{P}$ defines a family of affine cubic surfaces given by the equation $x_{1} x_{2} x_{3}-x_{1}-\alpha x_{2}-x_{3}+\alpha+1=0$. This surface is not singular for $\alpha \neq 1$. For $\alpha=1$ there is one singular point $\left(x_{1}, x_{2}, x_{3}\right)=(1,1,1)$ and this corresponds to the 'reducible locus' which, in terms of the above matrices, is the union of the two closed sets $\alpha=1, b_{1}=b_{3}=b_{5}=0$ and $\alpha=1, b_{2}=b_{4}=b_{6}=0$.

The results for this second family consist of the construction of fine moduli spaces $\mathcal{M}(\theta / 2)$ replacing the set $\mathbf{S}$. In each case, depending on $\theta$ with $e^{\pi i \theta}=\alpha$, the fine moduli space is identified with an Okamoto-Painlevé space. We note that the cases $\frac{\theta}{2} \in\{0,-1\}$ are rather subtle, since these involve reducible differential modules. As a corollary, one obtains the Painlevé property for every PII equation (in standard form). The rational solutions and the Riccati solutions follow from these moduli spaces. Moreover one obtains the Bäcklund transformations in a natural way as isomorphisms between the various moduli spaces. Finally, we discuss the relation between the two families $(0,-, 3 / 2)$ and $(-,-, 3)$.

1. The family $(0,-, 3 / 2)$. $\mathbf{S}$ is the set of the isomorphy classes of the differential modules of type $(0,-, 3 / 2)$ described in the Introduction. According to vdP-Sa], Theorem 1.7 , one has 
Proposition 1.1. The map $\mathbf{S} \rightarrow \mathcal{R} \times T$, which assigns to (an isomorphy class of) a module $M \in \mathbf{S}$ its three Stokes maps at $\infty$ and the $t \in T=\mathbb{C}$, which appears in the generalized eigenvalues $\pm\left(z^{3 / 2}+\frac{t}{2} z^{1 / 2}\right)$, is bijective.

We want to replace $M \in \mathbf{S}$ be a connection $(\mathcal{V}, \nabla)$ on the projective line with $\nabla$ : $\mathcal{V} \rightarrow \Omega([0]+3[\infty]) \otimes \mathcal{V}$ and $M$ is the generic fiber of $(\mathcal{V}, \nabla)$. Thus the given derivation $\partial$ on $M$ is the one induced by $\nabla_{\frac{d}{d z}}$ on the vector bundle $\mathcal{V}$. A choice of $(\mathcal{V}, \nabla)$ induces a $\mathbb{C}[[z]]$-lattice $\widehat{\mathcal{V}}_{0}$ in $\mathbb{C}((z)) \otimes M$ which is invariant under $z \partial$ and a $\mathbb{C}\left[\left[z^{-1}\right]\right]$-lattice in $\mathbb{C}\left(\left(z^{-1}\right)\right) \otimes M$ which is invariant under $z^{-1} \partial$. We will call these lattices invariant lattices.

On the other hand (see vdP-Sa] $\S 1.8$ or vdP-Si] p. 176), given an invariant lattice $\Lambda(0) \subset \mathbb{C}((z)) \otimes M$ (i.e., $(z \partial) \Lambda(0) \subset \Lambda(0))$ and an invariant lattice $\Lambda(\infty) \subset \mathbb{C}\left(\left(z^{-1}\right)\right) \otimes M$ (i.e., $\left.\left(z^{-1} \partial\right) \Lambda(\infty) \subset \Lambda(\infty)\right)$, there is a unique connection $(\mathcal{V}, \nabla)$ with:

- $\nabla: \mathcal{V} \rightarrow \Omega([0]+3[\infty]) \otimes \mathcal{V}$ has generic fiber $M$,

- $\left(\widehat{\mathcal{V}}_{0}, z \nabla_{\frac{d}{d z}}\right)$ is isomorphic to $(\Lambda(0), z \partial)$ and

- $\left(\widehat{\mathcal{V}}_{\infty}, z^{-1} \nabla_{\frac{d}{d z}}\right)$ is isomorphic to $\left(\Lambda(\infty), z^{-1} \partial\right)$.

The computation of the invariant lattices at $\infty$ is very similar to the one in $\mathrm{vdP}$, Proposition 1.1 (see also vdP-Sa $§ 1.2)$. We omit the proof.

Proposition 1.2. The invariant lattices of $N:=\mathbb{C}\left(\left(z^{-1}\right)\right) \otimes M$ are $\left\{N_{k}\right\}_{k \in \mathbb{Z}}$ with the properties:

(1) $N_{0}$ is represented for a suitable basis $f_{1}, f_{2}$ over $\mathbb{C}\left[\left[z^{-1}\right]\right]$ by the matrix operator

$$
\delta+\left(\begin{array}{cc}
1 / 4 & z^{-1 / 2} w \\
z^{1 / 2} w & -1 / 4
\end{array}\right), \quad \text { where } \delta:=z \frac{d}{d z} \text { and } w=z^{3 / 2}+\frac{t}{2} z^{1 / 2} .
$$

(2) $N_{-1}$ has basis $z^{-1} f_{1}, f_{2}$ and is represented by the matrix differential operator

$$
\delta+\left(\begin{array}{cc}
-1+1 / 4 & z^{1 / 2} w \\
z^{-1 / 2} w & -1 / 4
\end{array}\right)
$$

(3) $z^{k} N_{0}=N_{2 k}, z^{k} N_{-1}=N_{2 k-1}$ and $\Lambda^{2} N_{k}=\mathbb{C}\left[\left[z^{-1}\right]\right] b_{k}$ with $\delta\left(b_{k}\right)=k b_{k}$.

Let $N$ be a vector space over $\mathbb{C}((z))$ of dimension 2 . By $\left\langle e_{1}, e_{2}\right\rangle$ we denote the lattice in $N$ generated by $e_{1}, e_{2}$, i.e., $\left\langle e_{1}, e_{2}\right\rangle=\mathbb{C}[[z]] e_{1} \oplus \mathbb{C}[[z]] e_{2}$.

Proposition 1.3. Let $N$ be a regular singular differential module over $\mathbb{C}((z))$ with $\operatorname{dim} N=2$ and trivial $\Lambda^{2} N$. On $N$ we consider the operator $\delta=z \partial$. A $\mathbb{C}[[z]]$-lattice $\Lambda$ is called invariant if $\delta(\Lambda) \subset \Lambda$. The modules $N$ are classified by the conjugacy class of

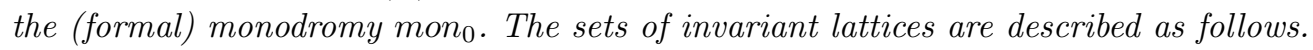

(1) Suppose mon $_{0}=\left(\begin{array}{cc}\beta & 0 \\ 0 & \frac{1}{\beta}\end{array}\right)$ with $\beta \neq 1,-1$. Then $\delta$ has the matrix $\left(\begin{array}{cc}\theta / 2 & 0 \\ 0 & -\theta / 2\end{array}\right)$ with $e^{\pi i \theta}=\beta$ on a certain basis $e_{1}, e_{2}$ of $N$. The invariant lattices are $\left\langle z^{n} e_{1}, z^{m} e_{2}\right\rangle$ with $m, n \in \mathbb{Z}$.

(2) Suppose mon $_{0}=\left(\begin{array}{ll}1 & 0 \\ 0 & 1\end{array}\right)$. Then $\delta e_{1}=\delta e_{2}=0$ for a certain basis $e_{1}, e_{2}$ of $N$. The invariant lattices are $\left\langle z^{n} a, z^{m} b\right\rangle$ with $n \leq m$ and $\{a, b\}$ a basis of $\mathbb{C} e_{1} \oplus \mathbb{C} e_{2}$. The lattice $\left\langle z^{n} a, z^{m} b\right\rangle$ is determined by $n \leq m$ and the line $\mathbb{C} a \subset \mathbb{C} e_{1} \oplus \mathbb{C} e_{2}$ in case $n<m$.

(3) Suppose mon $_{0}=\left(\begin{array}{ll}1 & x \\ 0 & 1\end{array}\right)$ with $x \neq 0$. Then $\delta$ has the form $\delta e_{1}=0, \delta e_{2}=y e_{1}, y \neq 0$ for a certain basis $e_{1}, e_{2}$ of $N$. The invariant lattices are $\left\langle z^{n} e_{1}, z^{m} e_{2}\right\rangle$ with $n, m \in \mathbb{Z}$ and $n \leq m$. 
(4) Suppose mon $_{0}=\left(\begin{array}{rr}-1 & 0 \\ 0 & -1\end{array}\right)$. Then $\delta$ has the form $\delta e_{1}=\frac{1}{2} e_{1}, \delta e_{2}=\frac{1}{2} e_{2}$ for a certain basis $e_{1}, e_{2}$ of $N$. The invariant lattices are $\left\langle z^{n} a, z^{m} b\right\rangle$ with $n \leq m$ and $\{a, b\}$ a basis of $\mathbb{C} e_{1} \oplus \mathbb{C} e_{2}$. The lattice $\left\langle z^{n} a, z^{m} b\right\rangle$ is determined by $n \leq m$ and the line $\mathbb{C} a \subset \mathbb{C} e_{1} \oplus \mathbb{C} e_{2}$ in case $n<m$.

(5) Suppose mon $_{0}=\left(\begin{array}{rr}-1 & x \\ 0 & -1\end{array}\right)$ with $x \neq 0$. Then $\delta$ has the form $\delta e_{1}=\frac{1}{2} e_{1}, \delta e_{2}=$ $\frac{1}{2} e_{1}+y e_{1}, y \neq 0$ for a certain basis $e_{1}, e_{2}$ of $N$. The invariant lattices are $\left\langle z^{n} e_{1}, z^{m} e_{2}\right\rangle$ with $n, m \in \mathbb{Z}$ and $n \leq m$.

Proof. (1) is easily verified. Further (4) and (5) follow easily from (2), (3).

(2) Consider an invariant lattice $\Lambda \subset \mathbb{C}((z)) e_{1} \oplus \mathbb{C}((z)) e_{2}$. Write a $\lambda \in \Lambda$ in the form $\lambda=\sum_{i \geq 0} a_{i} z^{i}$ with all $a_{i} \in \mathbb{C} e_{1}+\mathbb{C} e_{2}$. For any integer $k \geq 0$ we have $\delta^{k}(\lambda)=$ $\sum_{i>0} i^{k} a_{i} z^{i} \in \Lambda$. It follows that each term $a_{i} z^{i}$ belongs to $\Lambda$. Thus any $\Lambda$ is generated by elements of the form $a z^{i}$ with $a \in \mathbb{C} e_{1}+\mathbb{C} e_{2}$ and $i \in \mathbb{Z}$. One now easily verifies that the invariant lattices have the form $\left\langle a z^{n}, b z^{m}\right\rangle$ with $n \leq m$ and $\{a, b\}$ forms a basis of $V$. This lattice is uniquely determined by the integers $n \leq m$ and the line $\mathbb{C} a \subset \mathbb{C} e_{1}+\mathbb{C} e_{2}$ in case $n<m$.

(3) Let $\Lambda$ be an invariant lattice. After replacing $\Lambda$ by $z^{k} \Lambda$ for a suitable integer $k$ we may suppose that $\Lambda \subset\left\langle e_{1}, e_{2}\right\rangle$ and $\Lambda \not \subset z\left\langle e_{1}, e_{2}\right\rangle$. If $\Lambda \neq\left\langle e_{1}, e_{2}\right\rangle$, then $\Lambda \subset\left\langle e_{1}, z e_{2}\right\rangle$ because $\delta$ has on the $\mathbb{C}$-vector space $\left\langle e_{1}, e_{2}\right\rangle / z\left\langle e_{1}, e_{2}\right\rangle$ only one invariant line. If $\Lambda \neq\left\langle e_{1}, z e_{2}\right\rangle$, then $\Lambda \subset\left\langle e_{1}, z^{2} e_{2}\right\rangle$. Indeed, $\delta$ has on $\left\langle e_{1}, z e_{2}\right\rangle / z\left\langle e_{1}, z e_{2}\right\rangle$ two invariant lines and $\Lambda \not \subset$ $\left\langle z e_{1}, z e_{2}\right\rangle$. Repeating this reasoning one finds that $\Lambda=\left\langle e_{1}, z^{k} e_{2}\right\rangle$ for some integer $k \geq 0$. Thus we find that the invariant lattices are the $\left\langle z^{n} e_{1}, z^{m} e_{2}\right\rangle$, with $n, m \in \mathbb{Z}, n \leq m$.

1.1. The choice of the connection for $M \in \mathrm{S}$. Let $(\mathcal{W}, \nabla)$ be the connection corresponding to $M$, the lattice $N_{0}$ at $\infty$ and a lattice $L$ at 0 with $\Lambda^{2} L$ is trivial. The second exterior power of $(\mathcal{W}, \nabla)$ has no singularities and is therefore equal to $d: O \rightarrow \Omega$. Thus $\mathcal{W}$ has degree 0 and is equal to $O(k) \oplus O(-k)$ for some integer $k \geq 0$. Since the connection is irreducible, the defect, which is $k-(-k)$, is $\leq 2$. Thus $k=0$ or $k=1$. Let $(\mathcal{V}, \nabla)$ be the subconnection of $(\mathcal{W}, \nabla)$ given by the lattice $N_{-1}$ at $\infty$ and $L$ at 0 . Then $\mathcal{V} \cong O \oplus O(-1)$ and can be identified with $O e_{1} \oplus O(-[\infty]) e_{2}$.

The operator $\delta:=\nabla_{z \frac{d}{d z}}$ w.r.t. $e_{1}, e_{2}$ has the form $z \frac{d}{d z}+\left(\begin{array}{cc}a & b \\ c-a\end{array}\right)$ with $a=a_{0}+a_{1} z+$ $a_{2} z^{2}, b=b_{0}+b_{1} z+b_{2} z^{2}+b_{3} z^{3}, c=c_{0}+c_{1} z$. The condition at infinity is

$$
z \frac{d}{d z}+\left(\begin{array}{cc}
a & z^{-1} b \\
z c & -a-1
\end{array}\right)=U^{-1}\left(z \frac{d}{d z}+\left(\begin{array}{cc}
-1+1 / 4 & z^{1 / 2} w \\
z^{-1 / 2} w & -1 / 4
\end{array}\right)\right) U
$$

for some $U \in \operatorname{GL}\left(2, \mathbb{C}\left[\left[z^{-1}\right]\right]\right)$. This can be simplified to $a(a+1)+b c=w^{2}+r$ where $r$ is a polynomial of degree $\leq 1$ and is equivalent to the equations

$$
a_{2}^{2}+b_{3} c_{1}=0, \quad 2 a_{2} a_{1}+b_{3} c_{0}+b_{2} c_{1}=1, \quad 2 a_{0} a_{2}+a_{2}+a_{1}^{2}+b_{2} c_{0}+b_{1} c_{1}=t .
$$

Now we have to specify the choice of the lattice $L$ at 0 , or equivalently, the local equation for $\mathbb{C}((z)) \otimes M$ in matrix form. For the cases of Proposition 1.3 we obtain the following local equations.

(1) $z \frac{d}{d z}+\left(\begin{array}{lr}-\theta / 2 & 0 \\ 0 & \theta / 2\end{array}\right)$ and $\theta \notin \mathbb{Z}$.

(2) $z \frac{d}{d z}+\left(\begin{array}{cc}-m & 0 \\ 0 & m\end{array}\right)$ with $m \in \mathbb{Z}_{\geq 0}$.

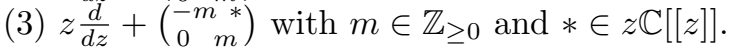


(4) $z \frac{d}{d z}+\left(\begin{array}{cc}-m-\frac{1}{2} & 0 \\ 0 & m+\frac{1}{2}\end{array}\right)$ with $m \in \mathbb{Z}_{\geq 0}$.

(5) $z \frac{d}{d z}+\left(\begin{array}{cc}-m-\frac{1}{2} & * \\ 0 & m+\frac{1}{2}\end{array}\right)$ with $m \in \mathbb{Z}_{\geq 0}$ and $* \in z \mathbb{C}[[z]]$.

Case (3) is rather subtle. The local equation has certainly the form $z \frac{d}{d z}+\left(\begin{array}{cc}-m+a & b \\ c & m-a\end{array}\right)$ with $a, b, c \in z \mathbb{C}[[z]]$. The equivalent equation:

$$
\left(\begin{array}{cc}
1 & 0 \\
-\alpha & 1
\end{array}\right)\left\{z \frac{d}{d z}+\left(\begin{array}{cc}
-m+a & b \\
c & m-a
\end{array}\right)\right\}\left(\begin{array}{ll}
1 & 0 \\
\alpha & 1
\end{array}\right)
$$

has the form $z \frac{d}{d z}+\left(\begin{array}{ll}A & B \\ C & D\end{array}\right)$ with $C=z \frac{d}{d z}(\alpha)+2 m \alpha-2 \alpha a-\alpha^{2} b+c$. Since the integer $m$ is $\geq 0$, there exists (a unique) $\alpha \in z \mathbb{C}[[z]]$ such that $C=0$. After that the transformation to $z \frac{d}{d z}+\left(\begin{array}{cc}-m * \\ 0 & m\end{array}\right)$ is easily found. The case (5) is similar.

The condition at 0 is

$$
z \frac{d}{d z}+\left(\begin{array}{cc}
a & b \\
c & -a
\end{array}\right)=U^{-1}\left\{z \frac{d}{d z}+\left(\begin{array}{cc}
-\theta / 2 & * \\
0 & \theta / 2
\end{array}\right)\right\} U
$$

for some $U \in \mathrm{GL}(2, \mathbb{C}[[z]])$ and $* \in \mathbb{C}[[z]]$. Equivalently $a_{0}^{2}+b_{0} c_{0}=\frac{\theta^{2}}{4}$. We note that in the case $\theta \in \mathbb{Z}$, we have normalized to $\theta \geq 0$.

The affine variety $\mathcal{A}$ given by the nine variables $a_{0}, \ldots, c_{1}$ and the above three equations has to be divided out by the automorphism group $G$ acting on $\mathcal{A}$ and defined by the base change $e_{1} \mapsto e_{1}, e_{2} \mapsto \lambda e_{2}+(\alpha+\beta z) e_{1}$ with $\alpha, \beta \in \mathbb{C}$ and $\lambda \in \mathbb{C}^{*}$.

Proposition 1.4. For any choice of $\theta$, the quotient $\mathcal{M}$ of $\mathcal{A}$ by the action of $G$ is a good quotient in the sense that there exists a $G$-equivariant isomorphism $G \times \mathcal{M} \rightarrow \mathcal{A}$. Further $\mathcal{M}$ is non-singular for $\theta \neq 0$.

Proof. $\mathcal{A}$ is the union of two open subsets, namely $\mathcal{A}\left(c_{0} \neq 0\right)$ and $\mathcal{A}\left(c_{1} \neq 0\right)$. For $\xi \in \mathcal{A}\left(c_{0} \neq 0\right)$ there exists a unique element $g \in G$ such that $g \xi$ is represented by the matrix differential operator

$$
z \frac{d}{d z}+\left(\begin{array}{cc}
a_{2} z^{2} & b_{0}+\cdots+b_{3} z^{3} \\
1+\frac{c_{1}}{c_{0}} z & -a_{2} z^{2}
\end{array}\right)
$$

where we have the equations

$$
a_{0}=a_{1}=0, \quad a_{2}^{2}+b_{3} \frac{c_{1}}{c_{0}}=0, \quad b_{3}+b_{2} \frac{c_{1}}{c_{0}}=1, \quad b_{0}=\frac{\theta^{2}}{4}, \quad a_{2}+b_{2}+b_{1} \frac{c_{1}}{c_{0}}=t .
$$

This defines a non-singular affine space $\mathcal{M}_{0}$ of dimension 3 with coordinate ring $\mathbb{C}\left[a_{2}, b_{2}, b_{1}, \frac{c_{1}}{c_{0}}\right] /\left(a_{2}^{2}+\left(1-b_{2} \frac{c_{1}}{c_{0}}\right) \frac{c_{1}}{c_{0}}\right)$.

For $\xi \in \mathcal{A}\left(c_{1} \neq 0\right)$, there is a unique $g \in G$ such that $g \xi$ is given by the matrix differential operator

$$
z \frac{d}{d z}+\left(\begin{array}{cc}
a_{0} & b_{0}+\cdots+b_{3} z^{3} \\
z+\frac{c_{0}}{c_{1}} & -a_{0}
\end{array}\right)
$$

where the equations are

$$
a_{1}=a_{2}=b_{3}=0, \quad b_{2}=1, \quad a_{0}^{2}+b_{0} \frac{c_{0}}{c_{1}}=\frac{\theta^{2}}{4}, \quad \frac{c_{0}}{c_{1}}+b_{1}=t .
$$


The coordinate ring of this affine space is $\mathbb{C}\left[a_{0}, b_{0}, b_{1}, \frac{c_{0}}{c_{1}}\right] /\left(a_{0}^{2}+b_{0} \frac{c_{0}}{c_{1}}-\frac{\theta^{2}}{4}\right)$. This affine space $\mathcal{M}_{1}$ is for $\theta \neq 0$ non-singular of dimension 3. For $\theta=0$ one has one singular line, namely $a_{0}=b_{0}=\frac{c_{0}}{c_{1}}=0$.

Now $\mathcal{M}$ is obtained by gluing $\mathcal{M}_{0}$ and $\mathcal{M}_{1}$ in the obvious way. The morphism $G \times$ $\mathcal{M} \rightarrow \mathcal{A}$, given by $(g, \xi) \mapsto g \xi$ is by construction an isomorphism.

Since $\mathcal{M}$ depends on $\theta^{2}$, we will write $\mathcal{M}\left(\theta^{2}\right)$ in the sequel. Write $q: \mathcal{M}\left(\theta^{2}\right) \rightarrow \mathbb{P}^{1}$ for the morphism $q(\xi)=-\frac{c_{1}}{c_{0}}$ in the notation of the proof of Proposition 1.4. The RiemannHilbert map $R H: \mathcal{M}\left(\theta^{2}\right) \rightarrow \mathcal{R}\left(e^{i \pi \theta}+e^{-i \pi \theta}\right)$ associates to a connection its analytic data and thus a point of $\mathcal{R}\left(e^{i \pi \theta}+e^{-i \pi \theta}\right)$. This map is known to be analytic. The fibers of the Riemann-Hilbert map $R H: \mathcal{M}\left(\theta^{2}\right) \rightarrow \mathcal{R}\left(e^{i \pi \theta}+e^{-i \pi \theta}\right)$ are, by definition, the isomonodromic families. These are parametrized by $t \in T=\mathbb{C}$. Let $q(t)$ denote the function $q$ restricted to an isomonodromic family. In vdP-Sa it is shown that $q=q(t)$ is a solution of the PII equation in Flaschka-Newell form (see also §2.2)

$$
q^{\prime \prime}=\frac{\left(q^{\prime}\right)^{2}}{2 q}+4 q^{2}+2 t q-\frac{\theta^{2}}{2 q} .
$$

1.2. The non-resonant case $\theta \notin \mathbb{Z}$. Let $R H^{+}=(R H, t): \mathcal{M}\left(\theta^{2}\right) \rightarrow \mathcal{R}\left(e^{i \pi \theta}+e^{-i \pi \theta}\right) \times$ $T$ with $T=\mathbb{C}$ denote the extended Riemann-Hilbert map. From Proposition 1.1, part (1) of Proposition 1.3 and the construction of $\mathcal{M}\left(\theta^{2}\right)$ one deduces that $R H^{+}$is bijective and therefore an analytic isomorphism (see $[\mathrm{KK}]$ ). As in $\mathrm{vdP}$ this has the following consequences.

TheOREM 1.5. Let $\theta \notin \mathbb{Z}$. Then $t: \mathcal{M}\left(\theta^{2}\right) \rightarrow T$ with its foliation given by the fibers of $R H: \mathcal{M}\left(\theta^{2}\right) \rightarrow \mathcal{R}\left(e^{i \pi \theta}+e^{-i \pi \theta}\right)$, i.e., the isomonodromic families, is isomorphic to the Okamoto-Painlevé space corresponding to the equation $q^{\prime \prime}=\frac{\left(q^{\prime}\right)^{2}}{2 q}+4 q^{2}+2 t q-\frac{\theta^{2}}{2 q}$. Moreover this equation has the Painlevé property.

1.3. The resonant case $\theta \in \mathbb{Z}, \theta>0$. We consider the case $\theta \in 2 \mathbb{Z}, \theta>0$. Then $s=2$. An element $(\mathcal{V}, \nabla) \in \mathcal{M}\left(\theta^{2}\right)$ has more information than the value of $t \in T$ and the Stokes matrices, i.e., a point of $\mathcal{R}(s=2)$. Namely, the local monodromy at $z=0$ has the form $z \frac{d}{d z}+\left(\begin{array}{cc}-\theta & * \\ 0 & \theta\end{array}\right)$ and there is given a uniquely determined 'eigenline', i.e., $\mathbb{C} e$ where $e \neq 0$ is an eigenvector for the eigenvalue $-\theta$ of the matrix differential operator.

This leads to the following definition of $\mathcal{R}^{+}(2)$. As mentioned in the introduction, the topological monodromy mon $_{0}$ at $z=0$ (or equivalently at $z=\infty$ ) is the product $\left(\begin{array}{cc}0 & -1 \\ 1 & 0\end{array}\right)\left(\begin{array}{cc}1 & 0 \\ a_{1} & 1\end{array}\right)\left(\begin{array}{cc}1 & a_{2} \\ 0 & 1\end{array}\right)\left(\begin{array}{cc}1 & 0 \\ a_{3} & 1\end{array}\right)$. In this case $a_{2} a_{2} a_{3}+a_{1}-a_{2}+a_{3}=-2$ and mon $_{0}$ has eigenvalues 1,1 and has an 'eigenline', i.e., an invariant line. A point of $\mathcal{R}^{+}(2)$ consists of the tuple $\left(a_{1}, a_{2}, a_{3}\right) \in \mathcal{R}$ and an 'eigenline'. Explicitly, consider the subspace of $\mathbb{P}^{1} \times \mathbb{A}^{3}$ given by the equations

$$
\begin{gathered}
-a_{1} a_{2} a_{3}-a_{1}+a_{2}-a_{3}=2 \text { and } \\
\left(1-a_{2}\right) y_{0}-\left(a_{2} a_{3}+1\right) y_{1}=0,\left(1+a_{1} a_{2}\right) y_{0}+\left(a_{2}-1\right) y_{1}=0,
\end{gathered}
$$

where $y_{0}, y_{1}$ are homogeneous coordinates of $\mathbb{P}^{1}$ and $a_{1}, a_{2}, a_{3}$ are coordinates for $\mathbb{A}^{3}$. The first equation is equivalent to mon $_{0}$ has trace 2 . The other equations mean that $\left(y_{0}, y_{1}\right) \neq 0$ is an eigenvector of mon $_{0}$ (for the eigenvalue 1$)$. This defines $\mathcal{R}^{+}(2)$. 
The canonical morphism $\mathcal{R}^{+}(2) \rightarrow \mathcal{R}(2)$ is the minimal resolution. Indeed, $\mathcal{R}^{+}(2)$ is non-singular, outside the singular point $\left(a_{1}, a_{2}, a_{3}\right)=(-1,1,-1)$ this map is an isomorphism (i.e., $\left.\operatorname{mon}_{0}=\left(\begin{array}{c}1 \neq 0 \\ 0\end{array} 1\right)\right)$ and the preimage of the singular point $(-1,1,-1)$ (i.e., mon $\left._{0}=\left(\begin{array}{ll}1 & 0 \\ 0 & 1\end{array}\right)\right)$ is isomorphic to $\mathbb{P}^{1}$.

The morphism $\mathcal{M}\left(\theta^{2}\right) \rightarrow \mathcal{R}^{+}(2)$ associates to $\xi=(\mathcal{V}, \nabla)$ the element $R H(\xi) \in \mathcal{R}$ and the "eigenline" for mon $_{0}$ which is the 'eigenline' of the local matrix $\left(\begin{array}{cc}-\theta & * \\ 0 & \theta\end{array}\right)$. The extended map $\mathcal{M}\left(\theta^{2}\right) \rightarrow \mathcal{R}^{+}(2) \times T$ is analytic and bijective and therefore an analytic isomorphism.

The case $\theta \in 1+2 \mathbb{Z}$ can be analyzed in the same way. This leads to the following result:

ThEOREM 1.6. Let $\theta \in 2 \mathbb{Z}, \theta>0$. Then $t: \mathcal{M}\left(\theta^{2}\right) \rightarrow T$ with its foliation given by the fibers of $R H: \mathcal{M}\left(\theta^{2}\right) \rightarrow \mathcal{R}^{+}(2)$, i.e., the isomonodromic families, is isomorphic to the Okamoto-Painlevé space corresponding to the equation $q^{\prime \prime}=\frac{\left(q^{\prime}\right)^{2}}{2 q}+4 q^{2}+2 t q-\frac{\theta^{2}}{2 q}$. Moreover this equation has the Painlevé property. For $\theta \in 1+2 \mathbb{Z}$ the same holds with $\mathcal{R}^{+}(2)$ replaced by $\mathcal{R}^{+}(-2)$.

1.4. The resonant case $\theta=0$. For $\theta=0$ another construction is needed. Let $M \in$ $\mathbf{S}(s=2)$. We choose at first the connection $(\mathcal{W}, \nabla)$ corresponding to the invariant lattice $N_{0}$ at $\infty$ and the invariant lattice $\mathcal{W}_{0}$ at 0 corresponding to the local equation $z \frac{d}{d z}+\left(\begin{array}{ll}0 & * \\ 0 & 0\end{array}\right)$. The action of $\delta$ on $\mathcal{W}_{0} / z \mathcal{W}_{0}$ is nilpotent (zero or not). We take a submodule $F:=\mathbb{C}\{z\} a$ with $\delta a=0$. Then $F+z \mathcal{W} \mathcal{W}_{0}$ is a new invariant lattice. Define now the connection $(\mathcal{V}, \nabla)$ by replacing at $z=0$, the invariant lattice $\mathcal{W}_{0}$ by $F+z \mathcal{W}_{0}$. As before, $\mathcal{V} \cong O \oplus O(-1)$ and can be represented as $O e_{1} \oplus O(-[0]) e_{2}$. Once again we compute the form of $\nabla$.

The operator $\delta:=\nabla_{z \frac{d}{d z}}$ has w.r.t. $e_{1}, e_{2}$ the form $z \frac{d}{d z}+\left(\begin{array}{cc}a & b \\ c & -a\end{array}\right)$ where

$$
a=a_{0}+a_{1} z+a_{2} z^{2}, \quad b=b_{-1} z^{-1}+b_{0}+\cdots+b_{2} z^{2}, \quad c=c_{1} z+c_{2} z^{2} .
$$

The condition at infinity is equivalent to $a^{2}+b c=w^{2}+r=z^{3}+t z^{2}+r$ where $r$ is a polynomial of degree $\leq 1$. One finds the equations

$$
a_{2}^{2}+b_{2} c_{2}=0, \quad 2 a_{1} a_{2}+b_{2} c_{1}+b_{1} c_{2}=1, \quad a_{1}^{2}+2 a_{0} a_{2}+b_{0} c_{2}+b_{1} c_{1}=t .
$$

The condition at $z=0$ can be stated as

$$
z \frac{d}{d z}+\left(\begin{array}{cc}
a & b_{-1}+b_{0} z+\cdots+b_{2} z^{2} \\
c_{1}+c_{2} z & -a+1
\end{array}\right)=U^{-1}\left(z \frac{d}{d z}+\left(\begin{array}{rr}
0 & * \\
0 & -1
\end{array}\right)\right) U,
$$

with some $* \in z \mathbb{C}[[z]]$ and some $U \in \mathrm{GL}(2, \mathbb{C}[[z]])$. This yields the equivalent relation $a_{0}\left(a_{0}-1\right)+c_{1} b_{-1}=0$. The group $G$ consisting of the base transformations $e_{1} \mapsto e_{1}, e_{2} \mapsto$ $\lambda e_{2}+\left(\alpha+\beta z^{-1}\right) e_{1}, \lambda \neq 0$, acts on the above set of variables and relations. There is a good quotient $\mathcal{M}$ which is the union of two open affine subsets $\mathcal{M}_{1}, \mathcal{M}_{2}$ corresponding to $c_{1} \neq 0$ and $c_{2} \neq 0$. We compute these two affine spaces, using the method of Proposition 1.4.

The $c_{1} \neq 0$ normalization is

$$
z \frac{d}{d z}+\left(\begin{array}{cc}
a_{2} z^{2} & b_{-1} z^{-1}+b_{0}+\cdots+b_{2} z^{2} \\
z+\frac{c_{2}}{c_{1}} z^{2} & -a_{2} z^{2}
\end{array}\right),
$$

with equations

$$
a_{0}=a_{1}=b_{-1}=0, \quad a_{2}^{2}+b_{2} \frac{c_{2}}{c_{1}}=0, \quad b_{2}+b_{1} \frac{c_{2}}{c_{1}}=1, \quad b_{0} \frac{c_{2}}{c_{1}}+b_{1}=t .
$$


The coordinate ring is

$$
\mathbb{C}\left[a_{2}, b_{0}, b_{1}, \frac{c_{2}}{c_{1}}\right] /\left(a_{2}^{2}+\left(1-b_{1} \frac{c_{2}}{c_{1}}\right) \frac{c_{2}}{c_{1}}\right) .
$$

Thus $\mathcal{M}_{1}$ is connected, non-singular of dimension 3 .

The $c_{2} \neq 0$ case has normalization

$$
z \frac{d}{d z}+\left(\begin{array}{cc}
a_{0} & b_{-1} z^{-1}+b_{0}+\cdots+b_{2} z^{2} \\
\frac{c_{1}}{c_{2}} z+z^{2} & -a_{0}
\end{array}\right),
$$

with equations

$$
a_{1}=a_{2}=b_{2}=0, \quad b_{1}=1, \quad b_{0}+b_{1} \frac{c_{1}}{c_{2}}=t, \quad a_{0}\left(a_{0}-1\right)+c_{1} b_{-1}=0 .
$$

The coordinate ring is

$$
\mathbb{C}\left[a_{0}, b_{-1}, b_{0}, \frac{c_{1}}{c_{2}}\right] /\left(a_{0}\left(a_{0}-1\right)+b_{-1} \frac{c_{1}}{c_{2}}\right) .
$$

Thus $\mathcal{M}_{2}$ is connected, non-singular of dimension 3 .

By gluing one obtains a non-singular connected variety $\mathcal{M}$ of dimension 3 . The map gen $: \mathcal{M} \rightarrow \mathbf{S}(s=2)$, which associates to $(\mathcal{V}, \nabla)$ its generic fiber, is surjective. For

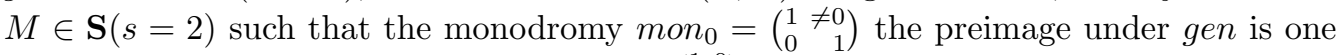
point. For $M \in \mathbf{S}(s=2)$ such that mon $_{0}=\left(\begin{array}{ll}1 & 0 \\ 0 & 1\end{array}\right)$, the preimage under gen is isomorphic to $\mathbb{P}^{1}$. Indeed, the elements in the preimage correspond, by part (2) of Proposition 1.3, to the invariant lattices $\langle a, z b\rangle$ and thus correspond to the lines $\mathbb{C} a \subset \mathbb{C} e_{1}+\mathbb{C} e_{2}$. One concludes that the natural analytic map $\mathcal{M} \rightarrow \mathcal{R}^{+}(s=2) \times T$ is bijection and thus is an analytic isomorphism. One finds

TheOREM 1.7. Let $\mathcal{M}$ be as above. Then $t: \mathcal{M} \rightarrow T$ with its foliation given by the fibers of $R H: \mathcal{M} \rightarrow \mathcal{R}^{+}(2)$, i.e., the isomonodromic families, is isomorphic to the OkamotoPainlevé space corresponding to the equation $q^{\prime \prime}=\frac{\left(q^{\prime}\right)^{2}}{2 q}+4 q^{2}+2 t q$. Moreover this equation has the Painlevé property.

2. The family $(-,-, 3)$. As in the Introduction, the set $\mathbf{S}$ consists of the isomorphy classes of the differential modules $M$ satisfying: $\operatorname{dim} M=2, \Lambda^{2} M$ is the trivial module, $\infty$ is the only singular point and $r(\infty)=3$, the generalized eigenvalues at $\infty$ are $\pm w$, $w=z^{3}+\frac{t}{2} z$. Further $\mathbf{S}(\alpha)$ denotes the subset of $\mathbf{S}$ consisting of the modules with $\alpha$ as eigenvalue of the formal monodromy. We start by computing connections with these data.

2.1. The moduli spaces $\mathcal{M}\left(\frac{\theta}{2}\right)$ of connections. We consider the connections $(\mathcal{V}, \nabla)$ with $\mathcal{V}=O e_{1}+O(-[\infty]) e_{2}, \nabla: \mathcal{V} \rightarrow \Omega(4[\infty]) \otimes \mathcal{V}$ and such that $\nabla_{z \frac{d}{d z}}$ is at $z=\infty$ isomorphic to the matrix differential operator $z \frac{d}{d z}+\left(\begin{array}{cc}\omega & 0 \\ 0 & -\omega-1\end{array}\right)$ with $\omega=z^{3}+\frac{t}{2} z+\frac{\theta}{2}$. The operator $D:=\nabla_{\frac{d}{d z}}$ has the form $\frac{d}{d z}+\left(\begin{array}{cc}a & b \\ c & -a\end{array}\right)$ with $a=a_{2} z^{2}+a_{1} z+a_{0}, b=b_{3} z^{3}+$ $\cdots+b_{0}, c=c_{1} z+c_{0}$. For the local basis $e_{1}, z^{-1} e_{2}$ the matrix of $z D$ is $\left(\begin{array}{cc}z a & b \\ z^{2} c & -z a-1\end{array}\right)$. The determinant of this matrix is equal to the determinant of $\left(\begin{array}{cc}\omega & 0 \\ 0 & -\omega-1\end{array}\right)$, moduli $z^{2} \mathbb{C}\left[\left[z^{-1}\right]\right]$. 
Thus $z a(z a+1)+z^{2} b c=\omega(\omega+1)+z^{2} \mathbb{C}\left[\left[z^{-1}\right]\right]$. This is equivalent to the equations

$$
\begin{gathered}
a_{2}^{2}+b_{3} c_{1}=1, \quad 2 a_{2} a_{1}+b_{3} c_{0}+b_{2} c_{1}=0, \quad 2 a_{2} a_{0}+a_{1}^{2}+b_{2} c_{0}+b_{1} c_{1}=t, \\
2 a_{1} a_{0}+a_{2}+b_{1} c_{0}+b_{0} c_{1}=\theta+1 .
\end{gathered}
$$

The above variables and equations define an affine variety $\mathcal{A}\left(\frac{\theta}{2}\right)$. The basis $e_{1}, e_{2}$ is unique up to a transformation $e_{1} \mapsto \lambda e_{1}, e_{2} \mapsto \mu e_{2}+\left(x_{0}+x_{1} z\right) e_{1}$ with $\lambda, \mu \in \mathbb{C}^{*}, x_{0}, x_{1} \in \mathbb{C}$. For its action on the matrix $\left(\begin{array}{cc}a & b \\ c & -a\end{array}\right)$, we may suppose $\lambda=1$. Let $G$ denote the group of transormations, given by $e_{1} \mapsto e_{1}, e_{1} \mapsto \mu e_{2}+\left(x_{0}+x_{1} z\right) e_{1}$. Then, in order to obtain a moduli space, we have to divide $\mathcal{A}\left(\frac{\theta}{2}\right)$ by the action of $G$. As we will show, this works well outside the subset of $\mathcal{A}\left(\frac{\theta}{2}\right)$ given by $c_{0}=c_{1}=0$.

For the subset of $\mathcal{A}\left(\frac{\theta}{2}\right)$, given by $c_{0}=c_{1}=0$, there are two possibilities:

$$
a_{2}=1, a_{1}=0, a_{0}=\frac{t}{2}, \theta=0 \quad \text { or } \quad a_{2}=-1, a_{1}=0, a_{0}=-\frac{t}{2}, \theta=-2 .
$$

In the first case, i.e. $\mathcal{A}(0)$, there is a unique element in $G$ which transforms any element $\frac{d}{d z}+\left(\begin{array}{cc}a & b \\ 0 & -a\end{array}\right)$ of this closed subset into $\frac{d}{d z}+\left(\begin{array}{cc}z^{2}+\frac{t}{2} & 0 \\ 0 & -z^{2}-\frac{t}{2}\end{array}\right)$. Thus, this closed subset consists of the connections which are a direct sum. Moreover this closed subset is one orbit under $G$.

The second case is similar. The closed subset of $\mathcal{A}(-1)$, given by $c_{0}=c_{1}=0$, consists of the connections which are direct sums and it is the orbit of $\frac{d}{d z}+\left(\begin{array}{cc}z^{2}+\frac{t}{2}-\frac{1}{z} & 0 \\ 0 & -z^{2}-\frac{t}{2}+\frac{1}{z}\end{array}\right)$ under $G$.

In the sequel we will avoid connections which are direct sums and write $\mathcal{A}^{*}\left(\frac{\theta}{2}\right)$ for the open subset of $\mathcal{A}\left(\frac{\theta}{2}\right)$ given by $\left(c_{0}, c_{1}\right) \neq(0,0)$. Thus $\mathcal{A}^{*}\left(\frac{\theta}{2}\right)=\mathcal{A}\left(\frac{\theta}{2}\right)$ except for $\frac{\theta}{2} \in\{0,-1\}$.

The space $\mathcal{A}^{*}\left(\frac{\theta}{2}\right)$ is the union of the two affine open subsets, given by $c_{1} \neq 0$ and $c_{0} \neq 0$. On the open subset, given by $c_{1} \neq 0$, one considers the closed subset $\mathcal{M}\left(\frac{\theta}{2}\right)_{1}$, defined by $a_{1}=a_{2}=0, c_{1}=1$. A computation (similar to the one in $\S 1$ and to computations in $\mathrm{vdP}$ ) shows that the map $G \times \mathcal{M}\left(\frac{\theta}{2}\right)_{1} \rightarrow \mathcal{A}^{*}\left(\frac{\theta}{2}\right)_{c_{1} \neq 0}$ is an isomorphism. The points of $\mathcal{M}\left(\frac{\theta}{2}\right)_{1}$ are

$$
\nabla_{\frac{d}{d z}}=\frac{d}{d z}+\left(\begin{array}{cc}
a_{0} & b \\
z-q & -a_{0}
\end{array}\right) \text { with } b=z^{3}+q z^{2}+\left(t+q^{2}\right) z+\theta+1+q\left(t+q^{2}\right) .
$$

Let $\mathcal{M}\left(\frac{\theta}{2}\right)_{0}$ denote the closed subset of $\mathcal{A}^{*}\left(\frac{\theta}{2}\right)_{c_{0} \neq 0}$, defined by $a_{0}=a_{1}=0, c_{0}=1$. Then $G \times \mathcal{M}\left(\frac{\theta}{2}\right)_{0} \rightarrow \mathcal{A}^{*}\left(\frac{\theta}{2}\right)_{c_{0} \neq 0}$ is an isomorphism. The points of $\mathcal{M}\left(\frac{\theta}{2}\right)_{0}$ are

$$
\begin{gathered}
\nabla_{\frac{d}{d z}}=\frac{d}{d z}+\left(\begin{array}{cc}
a_{2} z^{2} & b \\
c z+1 & -a_{0}
\end{array}\right) \text { with } b=b_{3} z^{3}+\cdots+b_{0} \text { and } \\
a_{2}^{2}+b_{3} c=1, \quad b_{3}+b_{2} c=0, \quad b_{2}+b_{1} c=t, \quad a_{2}+b_{1}+b_{0} c=\theta+1 .
\end{gathered}
$$

The moduli space $\mathcal{M}\left(\frac{\theta}{2}\right)$ is defined by gluing the two affine spaces $\mathcal{M}\left(\frac{\theta}{2}\right)_{1}$ and $\mathcal{M}\left(\frac{\theta}{2}\right)_{0}$ (over the open subsets $q \neq 0$ and $c \neq 0$ ) in the obvious way.

REMARKS 2.1. By construction $\mathcal{M}\left(\frac{\theta}{2}\right)$ is the good geometric quotient of $\mathcal{A}^{*}\left(\frac{\theta}{2}\right)$ by the action of $G$. One easily verifies that $\mathcal{M}\left(\frac{\theta}{2}\right)$ is a connected, non-singular variety of dimension 3. The fibers of the obvious morphism $t: \mathcal{M}\left(\frac{\theta}{2}\right) \rightarrow T=\mathbb{C}$ are non-singular and connected.

2.2. Isomonodromy. A family $\frac{d}{d z}+A$ with $A=\left(\begin{array}{cc}a & b \\ c & -a\end{array}\right)$ (in the space $\mathcal{M}\left(\frac{\theta}{2}\right)$ ), where the coefficients of $a, b, c$ are functions of $t \in T=\mathbb{C}$, is isomonodromic if and only if there 
is an operator $\frac{d}{d t}+B$ (the entries of the matrix $B$ depend in a polynomial way on $z$ and their coefficients are functions of $t$ ), commuting with $\frac{d}{d z}+\left(\begin{array}{cc}a & b \\ c & -a\end{array}\right)$. This is equivalent to $\frac{d}{d t}(A)=\frac{d}{d z}(B)+[A, B]$. For the computation we work on $\mathcal{M}\left(\frac{\theta}{2}\right)$ and since $B$ has also trace 0 , we will use the basis $H=\left(\begin{array}{cc}1 & 0 \\ 0 & -1\end{array}\right), E_{1}=\left(\begin{array}{ll}0 & 1 \\ 0 & 0\end{array}\right), E_{2}=\left(\begin{array}{ll}0 & 0 \\ 1 & 0\end{array}\right)$ of $\mathfrak{s l}_{2}$.

One writes $A=a H+b E_{1}+(z-q) E_{2}$ with $b=z^{3}+q z^{2}+\left(t+q^{2}\right) z+q\left(t+q^{2}\right)+\theta+1$ and $B=B_{H} H+B_{1} E_{1}+B_{2} E_{2}$ with $B_{*}=B_{*, 0}+B_{*, 1} z+B_{*, 2} z^{2}$ for $*=H, 1,2$ and all $B_{*, *}$ functions of $t$. Using the notation ${ }^{\prime}=\frac{d}{d t}$ and $o=\frac{d}{d z}$ one obtains the equations $a^{\prime}=\stackrel{o}{B}_{H}+b B_{2}-(z-q) B_{1}, b^{\prime}=\stackrel{o}{B}_{1}+2 a B_{1}-2 b B_{H},-q^{\prime}=\stackrel{o}{B}_{2}-2 a B_{2}+2(z-q) B_{H}$. A straightforward computation yields $q^{\prime}=a$ and $a^{\prime}=q\left(t+2 q^{2}\right)+\frac{\theta}{2}+\frac{1}{2}$. One concludes that the function $q=q(t)$ in the isomonodromic family satisfies the standard PII equation

$$
q^{\prime \prime}=2 q^{3}+t q+\frac{\theta}{2}+\frac{1}{2}
$$

We note that the differential equation in Theorems 1.5, 1.6, 1.7 can be obtained in the same way. For an isomonodromic family $z \frac{d}{d z}+\left(\begin{array}{cc}a & b \\ z-q & -a\end{array}\right)$ with $b=z^{2}+(t+q) z+$ $q^{-1}\left(a^{2}-\frac{\theta^{2}}{4}\right)$, one obtains the equations $q^{\prime}=2 a, a^{\prime}=q^{-1}\left(a^{2}-\frac{\theta^{2}}{4}\right)+q(t+2 q)$.

2.3. The monodromy space and $\mathcal{M}\left(\frac{\theta}{2}\right)$ for $\alpha \neq 1$. The monodromy data are given by the variables $\alpha, b_{1}, \ldots, b_{6}$ and the matrix equation

$$
\left(\begin{array}{cc}
\alpha & 0 \\
0 & \alpha^{-1}
\end{array}\right)\left(\begin{array}{ll}
1 & 0 \\
b_{1} & 1
\end{array}\right)\left(\begin{array}{cc}
1 & b_{2} \\
0 & 1
\end{array}\right) \cdots\left(\begin{array}{ll}
1 & b_{6} \\
0 & 1
\end{array}\right)=\left(\begin{array}{ll}
1 & 0 \\
0 & 1
\end{array}\right) .
$$

Fix $\alpha \neq 1$. There is no 1-dimensional subspace, invariant under all Stokes maps and the formal monodromy. One has to divide by the action (by conjugation) of the group $\mathbb{G}_{m}=\left\{\left(\begin{array}{ll}\lambda & 0 \\ 0 & 1\end{array}\right) \mid \lambda \in \mathbb{C}^{*}\right\}$. The result $\mathcal{R}(\alpha)$ is a geometric quotient and as a consequence the map $\mathbf{S}(\alpha) \rightarrow \mathcal{R}(\alpha) \times T$ is bijective.

Choose $\theta$ with $e^{\pi i \theta}=\alpha$. Let $M \in \mathbf{S}(\alpha)$ and consider the connection $(\mathcal{W}, \nabla)$ with the local matrix operator $z \frac{d}{d z}+\left(\begin{array}{cc}z^{3}+\frac{t}{2} z+\frac{\theta}{2} & 0 \\ 0 & -z^{3}-\frac{t}{2} z-\frac{\theta}{2}\end{array}\right)$ at $\infty$ and generic fiber $M$. Since the monodromy data for $M$ is irreducible, $M$ is irreducible, too. Then $\mathcal{W} \cong O(k) \oplus O(-k)$ with $k \in\{0,1\}$. Let $(\mathcal{V}, \nabla)$ be the sub-connection corresponding to the local operator $z \frac{d}{d z}+\left(\begin{array}{cc}w+\frac{\theta}{2} & 0 \\ 0 & -w-\frac{\theta}{2}-1\end{array}\right)$. Then $\mathcal{V} \cong O \oplus O(-1)$ and can be identified with $O e_{1}+O(-[\infty]) e_{2}$. It follows that the map $\mathcal{M}\left(\frac{\theta}{2}\right) \rightarrow \mathbf{S}(\alpha)$ is bijective. Therefore the extended Riemann-Hilbert map $\mathcal{M}\left(\frac{\theta}{2}\right) \rightarrow \mathcal{R}(\alpha) \times T$ is bijective. As in Section 1, one obtains:

Theorem 2.2. Suppose $\frac{\theta}{2} \notin \mathbb{Z}$. Then the PII equation in standard form $q^{\prime \prime}=2 q^{3}+$ $q t+\frac{\theta}{2}+\frac{1}{2}$ has the Painlevé property. The space $\mathcal{M}(\theta / 2)$ is the Okamoto-Painleve space associated to this PII equation.

2.4. The monodromy space and $\mathcal{M}\left(\frac{\theta}{2}\right)$ for $\alpha=1$. Now we consider $\mathbf{S}(1) \subset \mathbf{S}$ consisting of the modules with $\alpha=1$. The (naive) monodromy space $\mathcal{R}(1)$ is the categorical quotient of the subspace of $\mathbb{A}^{6}$ with variables $b_{1}, \ldots, b_{6}$ and equations given by

$$
\left(\begin{array}{ll}
1 & 0 \\
b_{1} & 1
\end{array}\right)\left(\begin{array}{ll}
1 & b_{2} \\
0 & 1
\end{array}\right) \cdots\left(\begin{array}{ll}
1 & b_{6} \\
0 & 1
\end{array}\right)=\left(\begin{array}{ll}
1 & 0 \\
0 & 1
\end{array}\right),
$$


divided out by the group $\mathbb{G}_{m}=\left\{\left(\begin{array}{cc}\lambda & 0 \\ 0 & 1\end{array}\right) \mid \lambda \in \mathbb{C}^{*}\right\}$, acting by conjugation. $\mathcal{R}(1)$ is the affine cubic surface given by the equation $x_{1} x_{2} x_{3}-x_{1}-x_{2}-x_{3}+2=0$ (the Cayley surface). The preimage of its unique singular point $\left(x_{1}, x_{2}, x_{3}\right)=(1,1,1)$ under the map $\mathbf{S}(1) \rightarrow \mathcal{R}(1)$ is the reducible locus of $\mathbf{S}(1)$ consisting of the reducible modules. In terms of the Stokes matrices, this preimage consists of the tuples $\left(b_{1}, \cdots, b_{6}\right)$ such that $b_{1}=b_{3}=b_{5}=0$ or $b_{2}=b_{4}=b_{6}=0$.

The map $\mathbf{S}(1) \rightarrow \mathcal{R}(1) \times T$ is surjective and not bijective since the categorical quotient $\mathcal{R}(1)$ is not a geometric quotient. A refinement of the categorical quotient and of $\mathbf{S}(1)$ is needed for the construction of moduli spaces of connections. We first study the reducible locus of $\mathbf{S}(1)$.

Lemma 2.3. Let $M \in \mathbf{S}(1)$ be reducible. Then one of the following holds.

(1) $M$ is a direct sum and has the form $\frac{d}{d z}+\left(\begin{array}{cc}\frac{w}{z} & 0 \\ 0 & -\frac{w}{z}\end{array}\right)$ (with $\left.w=z^{3}+\frac{t}{2} z\right)$

(2) $M$ has the form $\frac{d}{d z}+\left(\begin{array}{cc}\frac{w}{z} & 0 \\ c & -\frac{w}{z}\end{array}\right)$ and $c \neq 0$ has degree $\leq 1$ (the odd case)

(3) $M$ has the form $\frac{d}{d z}+\left(\begin{array}{cc}\frac{w}{z} & \begin{array}{c}z \\ 0\end{array} \\ 0 & -\frac{w}{z}\end{array}\right)$ and $b \neq 0$ has degree $\leq 1$ (the even case)

Moreover $c$ and $b$ are unique up to multiplication by a nonzero scalar.

Proof. Let $N$ be a 1-dimensional submodule of $M \in \mathbf{S}(1)$. Then $N$ has a singularity only at $\infty$ and thus has the operator form $\frac{d}{d z}+p$, where $p$ is a polynomial. The condition at $\infty$ implies that $z \frac{d}{d z}+(z p \pm w)$ is trivial over $\mathbb{C}\left(\left(z^{-1}\right)\right)$. Thus $p= \pm\left(z^{2}+\frac{t}{2}\right)$.

We consider the following case, which we will call "the odd case": For a certain basis $e_{1}, e_{2}$ of $M$ the operator $\partial$ has the form $D:=\frac{d}{d z}+\left(\begin{array}{cc}\frac{w}{z} & 0 \\ c & -\frac{w}{z}\end{array}\right)$ with $c \in \mathbb{C}(z)$. After a change of variables $e_{1} \mapsto e_{1}+h e_{2}, e_{2} \mapsto e_{2}$ and $h \in \mathbb{C}(z)$ one finds the same form with $c$ replaced by $\tilde{c}=c+h^{\prime}-2 \frac{w}{z} h$. One can choose $h$ such that $\tilde{c}$ has in $\mathbb{C}$ at most poles of order 1 . We continue with $c$ having at most poles of order 1 . The equation $D\left(\begin{array}{l}y_{1} \\ y_{2}\end{array}\right)=0$ has a basis of solutions $\left(e_{e^{\frac{z^{3}}{3}+\frac{t z}{2}}}^{0}\right)$ and $\left(\begin{array}{l}e^{-\frac{z^{3}}{3}-\frac{t z}{2}} \\ F e^{\frac{z^{3}}{3}+\frac{t z}{2}}\end{array}\right)$, where $F$ satisfies the equation $F^{\prime}=-c e^{-\frac{2}{3} z^{3}-t z}$. Since the above equation has two independent solutions on $\mathbb{C}$, the function $c$ has no poles of order one and thus $c \in \mathbb{C}[z]$. By the same transformation one finds a $c \in \mathbb{C}[z]$ of degree $\leq 1$. Let us write $D_{c}$ for this operator. The first possibility is $c=0$. We will show the following statement.

Suppose that $c \neq 0$ and that $D_{c}$ and $D_{\tilde{c}}$, for some $\tilde{c} \in \mathbb{C}[z]$ of degree $\leq 1$, are equivalent. Then $\tilde{c}=\lambda c$ for some $\lambda \in \mathbb{C}^{*}$.

Indeed, suppose that there is an $A=\left(\begin{array}{ll}a_{1} & a_{2} \\ a_{3} & a_{4}\end{array}\right) \in \mathrm{GL}(2, \mathbb{C}(z))$ such that $A D_{c}=D_{\tilde{c}} A$. Then one computes that $a_{2}=0, a_{1}, a_{4} \in \mathbb{C}^{*}$ and $a_{4} c=a_{3}^{\prime}-2 a_{3} \frac{w}{z}+a_{1} \tilde{c}$. Then $a_{3}$ has no poles. Since $c$ and $\tilde{c}$ have degree $\leq 1$ one finds that $a_{3}=0$ and the statement follows. This proves (2). The "even case" is similar.

The cases of Lemma 2.3 have the translation for the Stokes matrices:

(1) is equivalent to all $b_{i}=0$.

(2) is equivalent to $b_{2}=b_{4}=b_{6}=0$ and at least one of the $b_{1}, b_{3}, b_{5}$ is not 0 .

(3) is equivalent to $b_{1}=b_{3}=b_{5}=0$ and at least one of the $b_{2}, b_{4}, b_{6}$ is not 0 . 
The set $\mathbf{S}(1)$ is refined as follows: Case (1), the direct sum case, will be omitted. Further $\mathbf{S}_{i r r}(1)$ is the locus of the irreducible modules. $\mathbf{S}_{\text {odd }}(1)$ is the set of modules which are either irreducible or are reducible of type (2) of Lemma 2.3. Finally, $\mathbf{S}_{\text {even }}(1)$ is the set of modules which are either irreducible or are reducible of type (3) of Lemma 2.3.

The monodromy space is refined as follows: We omit the most singular case $b_{1}=\cdots=$ $b_{6}=0$. Let $\mathcal{R}_{i r r}(1)$ consists of the data $\left(b_{1}, \ldots, b_{6}\right)$ (with the relations) such that at least one of the $b_{1}, b_{3}, b_{5}$ is non-zero and also at least one of the $b_{2}, b_{4}, b_{6}$ is non-zero, divided by the group $\mathbb{G}_{m}$. This is a geometric quotient and thus $\mathbf{S}_{i r r}(1) \rightarrow \mathcal{R}_{i r r}(1) \times T$ is bijective.

$\mathcal{R}_{\text {odd }}(1)$ consists of the data $\left(b_{1}, \ldots, b_{6}\right)$ such that at least one of the $b_{1}, b_{3}, b_{5}$ is non-zero, divided by the group $\mathbb{G}_{m}$. This is a geometric quotient and the map $\mathbf{S}_{\text {odd }}(1) \rightarrow$ $\mathcal{R}_{\text {odd }}(1) \times T$ is bijective.

$\mathcal{R}_{\text {even }}(1)$ consists of the data $\left(b_{1}, \ldots, b_{6}\right)$ such that at least one of the $b_{2}, b_{4}, b_{6}$ is nonzero, divided by the group $\mathbb{G}_{m}$. This is a geometric quotient and the map $\mathbf{S}_{\text {even }}(1) \rightarrow$ $\mathcal{R}_{\text {even }}(1) \times T$ is bijective.

Proposition 2.4. Let $m:=\frac{\theta}{2} \in \mathbb{Z}$. The map $\mathcal{M}(m) \rightarrow \mathbf{S}(1)$, which associates to a connection its generic fiber, is injective. This map induces:

(1) for $m \neq 0,-1$, a bijection $\mathcal{M}(m) \rightarrow \mathbf{S}_{\text {irr }}(1)$;

(2) for $m=0$, a bijection $\mathcal{M}(0) \rightarrow \mathbf{S}_{\text {odd }}(1)$;

(3) for $m=-1$, a bijection $\mathcal{M}(-1) \rightarrow \mathbf{S}_{\text {even }}(1)$.

Proof. The map is injective since a connection in $\mathcal{M}(m)$ is determined by $M$ and the invariant lattice at $\infty$. Any $M \in \mathbf{S}_{\text {irr }}(1)$ lies in the image of $\mathcal{M}(m)$ for any $m \in \mathbb{Z}$. Using Lemma 2.3 and the explicit formulas for $\mathcal{A}(m)^{*}$ and $\mathcal{M}(m)$ one verifies that:

(1) $\frac{d}{d z}+\left(\begin{array}{cc}\frac{w}{z} & 0 \\ 0 & -\frac{w}{z}\end{array}\right)$ does not lie in the image of any $\mathcal{M}(m)$.

(2) $\frac{d}{d z}+\left(\begin{array}{cc}\frac{w}{z} & 0 \\ c & -\frac{w}{z}\end{array}\right)$ with $c \in \mathbb{C}[z], \operatorname{deg} c \leq 1, c \neq 0$, lies in the image of $\mathcal{M}(0)$ and does not lie in the image of $\mathcal{M}(m)$ with $m \neq 0$.

(3) $\frac{d}{d z}+\left(\begin{array}{cc}\frac{w}{z} & b \\ 0 & -\frac{w}{z}\end{array}\right)$ with $b \in \mathbb{C}[z], \operatorname{deg} b \leq 1, b \neq 0$, lies in the image of $\mathcal{M}(-1)$ and does not lie in the image of $\mathcal{M}(m)$ with $m \neq-1$.

Proposition 2.4 and the bijections $\mathbf{S}_{i r r}(1) \rightarrow \mathcal{R}_{i r r}(1) \times T, \mathbf{S}_{\text {odd }}(1) \rightarrow \mathcal{R}_{\text {odd }}(1) \times T$ and $\mathbf{S}_{\text {even }}(1) \rightarrow \mathcal{R}_{\text {even }}(1) \times T$, lead to analytic isomorphisms

- $R H^{+}: \mathcal{M}(m) \rightarrow \mathcal{R}_{i r r}(1) \times T$ for $m \neq 0,-1$,

- $R H^{+}: \mathcal{M}(0) \rightarrow \mathcal{R}_{\text {odd }}(1) \times T$ for $m=0$ and

- $R H^{+}: \mathcal{M}(-1) \rightarrow \mathcal{R}_{\text {even }}(1) \times T$ for $m=-1$.

As before, $q: \mathcal{M}(m) \rightarrow \mathbb{P}^{1}$ is defined by $q=-\frac{c_{0}}{c_{1}}$ and the restriction of $q$ to an isomonodromic family in $\mathcal{M}(m)$ satisfies, according to the computation in $\S 2.2$, the equation $q^{\prime \prime}=2 q^{3}+q t+m+\frac{1}{2}$. This leads to the following result.

Theorem 2.5. Let $m:=\frac{\theta}{2} \in \mathbb{Z}$. The equation $q^{\prime \prime}=2 q^{3}+q t+m+\frac{1}{2}$ has the Painlevé property. Moreover, $\mathcal{M}(m)$, provided with the projection $t: \mathcal{M}(m) \rightarrow T$ and the foliation given by $\left\{\left(R H^{+}\right)^{-1}(\{r\} \times T) \mid r\right\}$ coincides with the Okamoto-Painleve space for this PII equation. 
Remark on the Painlevé property. For the convenience of the reader we give the proof of the Painlevé property for the equation $q^{\prime \prime}=2 q^{3}+q t+\frac{\theta}{2}+\frac{1}{2}$. Let $Q$ denote a meromorphic solution of this equation in the neighbourhood of $t_{0}$. After shifting $t_{0}$ we may suppose that $Q$ is holomorphic on some open disk $D:=\left\{t \in \mathbb{C}|| t-t_{0} \mid<\epsilon\right\}$. We may suppose that $Q$ is not constant. Consider the map

$$
\begin{gathered}
f: t \in D \mapsto \frac{d}{d z}+\left(\begin{array}{cc}
a & b \\
z-Q(t) & -a
\end{array}\right), \\
b=z^{3}+Q(t) z^{2}+\left(t+Q(t)^{2}\right) z+Q(t)\left(t+Q(t)^{2}\right)+\theta+1
\end{gathered}
$$

and $a=Q^{\prime}(t)$. The formula in $\S 2.1$ shows that this matrix differential operator is not a direct sum. Thus $f$ is an analytic map $D \rightarrow \mathcal{M}\left(\frac{\theta}{2}\right)$. There is an analytic isomorphism $\mathcal{M}\left(\frac{\theta}{2}\right) \rightarrow \mathcal{R}(\alpha)_{*} \times T$, where $*=\operatorname{irr}$ for $\theta \neq 0,-1, *=$ odd for $\theta=0$ and $*=$ even for $\theta=-1$. Since $Q$ is a solution of PII, the formulas from $\S 2.2$ imply that the composed map $D \rightarrow \mathcal{M}\left(\frac{\theta}{2}\right) \rightarrow \mathcal{R}(\alpha)_{*}$ is constant. Hence $Q$ extends to a meromorphic function on $T=\mathbb{C}$.

\section{Special features of the family $(-,-, 3)$}

Riccati solutions of PII. Let $\mathcal{M}(0)_{\text {red }} \subset \mathcal{M}(0)$ denote the subspace of the reducible connections. By Lemma 2.3, the points of $\mathcal{M}(0)_{\text {red }}$ are represented by $\frac{d}{d z}+\left(\begin{array}{c}-\frac{w}{z} \\ c^{\frac{w}{z}}\end{array}\right)$ with $c=c_{1} z+c_{0} \neq 0$ (unique up to multiplication by a scalar). Thus $\mathcal{M}(0)_{\text {red }} \cong \mathbb{P}^{1} \times T$. The reducible locus $\mathcal{R}_{\text {odd }}(1)_{\text {red }}$ of $\mathcal{R}_{\text {odd }}(1)$ consists of the tuples $\left(b_{1}, b_{3}, b_{5}\right) \neq 0$ with $b_{1}+b_{3}+b_{5}=0$ modulo the action of multiplication by $\mathbb{C}^{*}$. This space is again a $\mathbb{P}^{1}$. Moreover, we have an analytic isomorphism $\mathcal{M}(0)_{\text {red }} \rightarrow \mathcal{R}_{\text {odd }}(1)_{\text {red }} \times T$, which is (after suitable identification) the identity map $\mathbb{P}^{1} \times T \rightarrow \mathbb{P}^{1} \times T$.

Now we study the isomonodromic families in $\mathcal{M}(0)_{\text {red }}$. One can normalize such a family as $\frac{d}{d z}+\left(\begin{array}{cc}-\frac{w}{z} & 0 \\ z-q & \frac{w}{z}\end{array}\right)$, where $q$ is a function of $t$. This operator has to commute (see vdP-Sa] $\S 4.1)$ with an operator of the form $\frac{d}{d t}+\left(\begin{array}{cc}b_{1} & 0 \\ b_{2} & -b_{1}\end{array}\right)$, where $b_{1}, b_{2}$ are polynomials in $z$ and with coefficients depending on $t$. An easy computation shows that $b_{1}=-(z+q) / 2, b_{2}=1 / 2$ and $q^{2}-q^{\prime}+t / 2=0$. This is the Riccati equation associated to the linear equation $y^{\prime \prime}+\frac{t}{2} y=0$ (by the substitution $q=-\frac{y^{\prime}}{y}$ ), which is essentially the Airy equation. Let $y_{1}, y_{2}$ be two independent solutions of this equation (holomorphic on $\mathbb{C}$ ). Then the solutions $q$ have the form $\left\{\frac{c_{1} y_{1}^{\prime}+c_{2} y_{2}^{\prime}}{c_{1} y_{1}+c_{2} y_{2}} \mid\left(c_{1}: c_{2}\right) \in \mathbb{P}^{1}\right\}$.

Using $\mathcal{M}(-1)_{\text {red }}$ one obtains in a similar way the Riccati equation $q^{2}+q^{\prime}+\frac{t}{2}=0$ and the corresponding $\mathbb{P}^{1}$-family of Riccati solutions.

Bäcklund transformations. Let $\mathcal{M}(\theta / 2)_{i r r}$ be the subspace corresponding to $\mathbf{S}\left(e^{i \pi \theta}\right)_{\text {irr }}$. For $\theta / 2 \neq 0,-1$, one has $\mathcal{M}(\theta / 2)_{i r r}=\mathcal{M}(\theta / 2)$ and for $\theta / 2=0,-1$ the complement of this Zariski open subset will be denoted by $\mathcal{M}(0)_{\text {red }}$ and $\mathcal{M}(-1)_{\text {red }}$.

The isomorphisms $\mathcal{M}(\theta / 2)_{i r r} \rightarrow \mathcal{R}(\alpha)_{i r r} \times T$ and $\mathcal{M}(1+\theta / 2)_{i r r} \rightarrow \mathcal{R}(\alpha)_{i r r} \times T$ (with $\alpha=e^{\pi i \theta}$ ) induce an isomorphism of the algebraic varieties $f(\theta): \mathcal{M}(\theta / 2)_{i r r} \rightarrow$ $\mathcal{M}(1+\theta / 2)_{i r r}$. This morphism commutes with the Riemann-Hilbert map and thus $f(\theta)$ maps isomonodromic families to isomonodromic families. In particular, $f(\theta)$ provides a map from the solutions $q$ of the equation $q^{\prime \prime}=2 q^{3}+q t+\frac{\theta}{2}+\frac{1}{2}$ to the solutions $Q$ of the 
equation $Q^{\prime \prime}=2 Q^{3}+Q t+1+\frac{\theta}{2}+\frac{1}{2}$, given by the formula $Q=-q-\frac{\theta+2}{2 q^{2}+2 q^{\prime}+t}$. The inverse of $f(\theta)$ has a similar formula $q \mapsto-q-\frac{\theta+2}{2 q^{2}-2 q^{\prime}+t}$.

Now $2 q^{2} \pm 2 q^{\prime}+t \neq 0$ follows from the fact that we work with the irreducible locus of the space $\mathcal{M}(\theta / 2)$ (see Riccati solutions of $\mathrm{PII})$.

The map $z \mapsto-z$, sends, according to the explicit family in $\S 2.1$, the points of $\mathcal{M}(\theta / 2)$ to the points of $\mathcal{M}(-1-\theta / 2)$. This is an isomorphism commuting with the RiemannHilbert map. The corresponding map from the solutions of $q^{\prime \prime}=2 q^{3}+q t+\frac{\theta}{2}+\frac{1}{2}$ to the solutions of $q^{\prime \prime}=2 q^{3}+q t-\frac{\theta}{2}-\frac{1}{2}$, is the obvious one $q \mapsto-q$. The two operations above generate the Bäcklund transformations for PII, which is the affine Weyl group of type $A_{1}$.

The algebraic solutions of PII in standard form $q^{\prime \prime}=2 q^{3}+t q+\frac{\theta}{2}+\frac{1}{2}$. Let $q$ be an algebraic solution of PII. At a suitable point $t_{0} \in T=\mathbb{C}$, a branch of $q$ is a local solution. Since the Painlevé property holds, this local solution extends to all of $T$. Thus the Riemann surface of $q$ has at most one branch point and therefore $q$ is rational. We conclude that the algebraic solutions are rational.

For any pole $t_{0} \in T$ of a rational solution $q$ of PII, the local expansion of $q$ is $q=$ $c_{-1}\left(t_{0}\right)\left(t-t_{0}\right)^{-1}+*+*\left(t-t_{0}\right)+\cdots$ with $c_{-1}\left(t_{0}\right)^{2}=1$.

If $q \neq 0$, then its expansion at $\infty$ has the form $c_{-1} t^{-1}+* t^{-2}+\cdots$ with $c_{-1} \neq 0$ and $\frac{\theta}{2}+\frac{1}{2}=-c_{-1}$. Now $q=\sum_{t_{0}} \frac{c_{-1}\left(t_{0}\right)}{t-t_{0}}$, where the sum is taken over some finite subset of $T=\mathbb{C}$ and $c_{-1}\left(t_{0}\right) \in\{-1,1\}$ for all $t_{0}$. It follows that $c_{-1}=\sum c_{-1}\left(t_{0}\right) \in \mathbb{Z}$ and $\frac{\theta}{2}+\frac{1}{2} \in \mathbb{Z}, \frac{\theta}{2}+\frac{1}{2} \neq 0$.

The remaining case $q=0$ gives 0 as the only algebraic solution of PII with $\theta=-1$. The equation $q^{\prime \prime}=2 q^{3}+t q$ corresponds to $\mathcal{M}(-1 / 2)$. By the above Bäcklund transformations one finds a single rational solution for $\mathcal{M}\left(n-\frac{1}{2}\right)$ and equation $q^{\prime \prime}=2 q^{3}+t q+n$ for every $n \in \mathbb{Z}$.

New transcendental solutions. In O3 the possible principal differential ideals of the differential algebra $\mathbb{C}(t)\left[q, q_{1}\right]$, given by $t^{\prime}=1, q^{\prime}=q_{1}, q_{1}^{\prime}=2 q^{3}+t q+\frac{\theta}{2}+\frac{1}{2}$, are computed. It suffices to consider ideals generated by a non-zero prime element $F$. Reconsidering this computation one finds (slightly different from [O3]) as the only possibilities $\frac{\theta}{2}=-1$, $F=q_{1}+q^{2}+t / 2$ and $\frac{\theta}{2}=0, F=q_{1}-q^{2}-t / 2$. All other solutions of PII are 'new' transcendental functions.

4. Comparing $(0,-, 3 / 2)$ and $(-,-, 3)$. The well known comparison between the corresponding equations is as follows. Let $q$ be a solution of the standard PII equation $q^{\prime \prime}=2 q^{3}+t q+\frac{\theta}{2}+\frac{1}{2}$. Define $p$ by $q^{\prime}=p-q^{2}-\frac{t}{2}$. One finds $p^{\prime}=2 p q+\frac{\theta}{2}+1$ and the Flaschka-Newell equation $p^{\prime \prime}=\frac{\left(p^{\prime}\right)^{2}}{2 p}+2 p^{2}-t p-\frac{\left(1+\frac{\theta}{2}\right)^{2}}{2 p}$.

If $p \neq 0$ is a solution of the Flaschka-Newell equation, then $q=\frac{p^{\prime}-\frac{\theta}{2}-1}{2 p}$ is a solution of the standard PII equation. For the special case $\frac{\theta}{2}=-1$, the Riccati solutions are mapped to the rational solution $p=0$.

There is a similar transformation to a Flaschka-Newell equation with $p$ defined by $q^{\prime}=p+q^{2}+\frac{t}{2}$. Now $p^{\prime}=-2 p q+\frac{\theta}{2}$ and $p^{\prime \prime}=\frac{\left(p^{\prime}\right)^{2}}{2 p}-2 p^{2}-p t-\frac{\left(\frac{\theta}{2}\right)^{2}}{2 p}$. If $p \neq 0$ is a solution, 
then $q=-\frac{p^{\prime}-\frac{\theta}{2}}{2 p}$ is a solution of the standard equation PII. For the special case $\frac{\theta}{2}=0$, the Riccati solutions are mapped to the rational solution $p=0$.

We note that the Bäcklund transformations for the Flaschka-Newell equation can be derived form these for the standard PII.

At present we do not know the full geometric interpretation of the above transformation. A partial result is the following. Let the differential module $M$ belong to the class $(0,-, 3 / 2)$ such that its topological monodromy at $z=0$ is $\pm\left(\begin{array}{ll}1 & 0 \\ 0 & 1\end{array}\right)$ (i.e., $\left.s= \pm 2\right)$. Then $N:=\mathbb{C}(u) \otimes M$, where $z=u^{2}$, belongs to the class $(-,-, 3)$ with $\alpha=-1$, or, in other words, the formal monodromy at $u=\infty$ is $\left(\begin{array}{cc}-1 & 0 \\ 0 & -1\end{array}\right)$.

Indeed, the monodromy data for $M($ at $z=\infty)$ are given by the formal monodromy and the three Stokes matrices

$$
\left(\begin{array}{cc}
0 & -1 \\
1 & 0
\end{array}\right),\left(\begin{array}{ll}
1 & 0 \\
b_{1} & 1
\end{array}\right),\left(\begin{array}{ll}
1 & b_{2} \\
0 & 1
\end{array}\right),\left(\begin{array}{ll}
1 & 0 \\
b_{3} & 1
\end{array}\right) .
$$

By assumption, their product is $\pm\left(\begin{array}{ll}1 & 0 \\ 0 & 1\end{array}\right)$. A computation, using the classification of $\mathrm{vdP}-\mathrm{Si}$ $\S 9.2$, shows that the monodromy data for $N$ at $u=\infty$, given by the formal monodromy and the six Stokes matrices, is

$$
\left(\begin{array}{cc}
-1 & 0 \\
0 & -1
\end{array}\right),\left(\begin{array}{ll}
1 & 0 \\
b_{1} & 1
\end{array}\right),\left(\begin{array}{ll}
1 & b_{2} \\
0 & 1
\end{array}\right),\left(\begin{array}{ll}
1 & 0 \\
b_{3} & 1
\end{array}\right),\left(\begin{array}{cc}
1 & -b_{1} \\
0 & 1
\end{array}\right),\left(\begin{array}{cc}
1 & 0 \\
-b_{2} & 1
\end{array}\right),\left(\begin{array}{cc}
1 & -b_{3} \\
0 & 1
\end{array}\right)
$$

and their product is $\left(\begin{array}{ll}1 & 0 \\ 0 & 1\end{array}\right)$.

An isomonodromic family of connections for the case $(0,-, 3 / 2)$ and $s= \pm 2$ yields (see Thm 1.6 and Thm 1.7) a solution $q$ for $q^{\prime \prime}=\frac{\left(q^{\prime}\right)^{2}}{2 q}+4 q^{2}+2 t q-\frac{\theta^{2}}{2 q}$ with $\theta \in \mathbb{Z}$. The morphism $\mathbb{P}^{1} \rightarrow \mathbb{P}^{1}$, corresponding to $\mathbb{C}(z) \subset \mathbb{C}(u)$ with $z=u^{2}$, induced an isomonodromic family for $(-,-, 3)$ with $\alpha=-1$. This produces a solution $q$ of $q^{\prime \prime}=2 q^{3}+t q+\theta$. This coincides (after normalization) with the above transformation from the Flaschka-Newell form to the standard form of PII.

Acknowledgments. The author thanks Masa-Hiko Saito for organizing his visit to the Department of Mathematics of the University of Kobe. Thanks are also due to Jaap Top and Masa-Hiko Saito for helpful discussions on the subject of this paper.

\section{References}

[B] P. Boutroux, Recherches sur les transcendantes de M. Painlevé et l'étude asymptotique des équations du second ordre, Ann. Sci. École. Norm. Sup. (3) 30 (1913), 255-375; suite 31 (1914), 99-159.

[Boa] P. Boalch, From Klein to Painlevé via Fourier, Laplace and Jimbo, Proc. London Math. Soc. (3) 90 (2005), 167-208.

[D-J] J. J. Duistermaat and N. Joshi, Okamoto's space for the first Painlevé equation in Boutroux coordinates, arXiv:1010.5563v I [math.CA] 27 Oct. 2010

[In] M. Inaba, Moduli of parabolic connections on a curve and Riemann-Hilbert correspondence, arXiv:math/0602004.

[IIS1] M. Inaba, K. Iwasaki and M.-H. Saito, Moduli of stable parabolic connections, Riemann-Hilbert correspondence and geometry of Painlevé equation of type VI. I, Publ. Res. Inst. Math. Sci. 42 (2006), 987-1089. 
[IIS2] M. Inaba, K. Iwasaki and M.-H. Saito, Moduli of stableparabolic connections, Riemann-Hilbert correspondence and geometry of Painlevé equation of type VI. II, in: Moduli Spaces and Arithmetic Geometry (Tokyo), Adv. Stud. Pure Math. 45, Math. Soc. Japan, Tokyo, 2006, 387-432.

[IISA] M. Inaba, K. Iwasaki and M.-H. Saito, Dynamics of the sixth Painlevé Equations, in: Théories Asymptotiques et Équations de Painlevé, Angers, Sém. Congrès 14, Société Mathématique de France, 2006, 103-167.

[JMU] M. Jimbo, T. Miwa and K. Ueno, Monodromy preserving deformation of linear ordinary differential equations with rational coefficients. I. General theory and $\tau$-function, Physica D 2 (1981), 306-352.

[JM] M. Jimbo and T. Miwa, Monodromy preserving deformation of linear ordinary differential equations with rational coefficients. II., Physica D 2 (1981), 407-448.

[KK] L. Kaup and B. Kaup, Holomorphic Functions of Several Variables, Walter de Gruyter, Berlin-New York, 1983.

[OKSO] Y. Ohyama, H. Kawamuko, H. Sakai and K. Okamoto, Studies on the Painlevé equations. V. Third Painlevé equations of special type $P_{\mathrm{III}}\left(D_{7}\right)$ and $P_{\mathrm{III}}\left(D_{8}\right)$, J. Math. Sci. Univ. Tokyo 13 (2006), 145-204.

[OO] Y. Ohyama and S. Okumura, A coalescent diagram of the Painlevé equations from the viewpoint of isomonodromic deformations, J. Phys. A 39 (2006), 12129-12151.

[O1] K. Okamoto, Sur les feuilletages associés aux équations du second ordre à points critiques fixes de P. Painlevé, Espaces des conditions initiales, Japan. J. Math. 5 (1979), 1-79.

[O2] K. Okamoto, Isomonodromic deformation and Painlevé equations and the Garnier system, J. Fac. Sci. Univ. Tokyo, Sect. IA Math. 33 (1986), 575-618.

[O3] K. Okamoto, The Hamiltonians associated to the Painlevé equations. The Painlevé property, in: CRM Ser. Math. Phys., Springer, New York, 1999, 735-787.

[vdP] M. van der Put, Families of linear differential equations and the Painlevé equations, preprint, 2011.

[vdP-Si] M. van der Put and M. F. Singer, Galois Theory of Linear Differential Equations, Grundlehren der mathematischen Wissenschaften 328, Springer, 2003.

[vdP-Sa] M. van der Put and M-H. Saito, Moduli spaces for linear differential equations and the Painlevé equations, Ann. Inst. Fourier 59 (2009), 2611-2667.

[STT] M.-H. Saito, T. Takebe and H. Terajima, Deformation of Okamoto-Painlevé pairs and Painlevé equations, J. Algebraic Geom. 11 (2002), 311-362.

[S-Ta] M.-H. Saito and T. Takebe, Classification of Okamoto-Painlevé pairs, Kobe J. Math. 19 (2002), 21-50.

[SU] M.-H. Saito and H. Umemura, Painlevé equations and deformations of rational surfaces with rational double points, in: Physics and Combinatorics 1999 (Nagoya), World Sci., River Edge, NJ, 2001, 320-365.

[STe] M.-H. Saito and H. Terajima, Nodal curves and Riccati solutions of Painlevé equations, J. Math. Kyoto Univ. 44 (2004), 529-568.

[Sakai] H. Sakai, Rational surfaces associated with affine root systems and geometry of the Painlevé equations, Comm. Math. Phys. 220 (2001), 165-229.

[T] H. Terajima, Families of Okamoto-Painlevé pairs and Painlevé equations, Ann. Mat. Pura Appl. (4) 186 (2007), 99-146. 